\title{
Timing of young radio pulsars II. Braking indices and their interpretation
}

\author{
A. Parthasarathy ${ }^{1,2,3,4},{ }^{\star}$ S. Johnston ${ }^{3}$, R.M. Shannon ${ }^{1,2}$, L. Lentati $^{5}$, M. Bailes $^{1,2}$, \\ S. Dai ${ }^{3}$, M. Kerr ${ }^{6}$, R.N. Manchester ${ }^{3}$, S. Osłowski1 ${ }^{1,2}$, C. Sobey ${ }^{7}$, \\ W. van Straten ${ }^{8}$, P. Weltevrede ${ }^{9}$ \\ 1 Centre for Astrophysics and Supercomputing, Swinburne University of Technology, P.O. Box 218, Hawthorn, Victoria 3122, Australia \\ 2 OzGrav: Australian Research Council Centre of Excellence for Gravitational Wave Discovery. \\ 3 CSIRO Astronomy and Space Science, Australia Telescope National Facility, PO Box 76, Epping NSW 1710, Australia \\ 4 Max-Planck-Institut für Radioastronomie, Auf dem Hügel 69, D-53121 Bonn, Germany \\ 5 Astrophysics Group, Cavendish Laboratory, JJ Thomson Avenue, Cambridge, CB3 OHE, UK \\ 6 Space Science Division, Naval Research Laboratory, Washington, DC 20375, USA \\ 7 CSIRO Astronomy and Space Science, PO Box 1130 Bentley, WA 6102, Australia \\ 8 Institute for Radio Astronomy 8 Space Research, Auckland University of Technology, Private Bag 92006, Auckland 1142, New Zealand \\ 9 Jodrell Bank Centre for Astrophysics, The University of Manchester, Alan Turing Building, Manchester, M13 9PL, United Kingdom
}

Accepted XXX. Received YYY; in original form ZZZ

\begin{abstract}
In Paper I of this series, we detected a significant value of the braking index $(n)$ for 19 young, high- $\dot{E}$ radio pulsars using $\sim 10$ years of timing observations from the $64-\mathrm{m}$ Parkes radio telescope. Here we investigate this result in more detail using a Bayesian pulsar timing framework to model timing noise and to perform selection to distinguish between models containing exponential glitch recovery and braking index signatures. We show that consistent values of $n$ are maintained with the addition of substantial archival data, even in the presence of glitches. We provide strong arguments that our measurements are unlikely due to exponential recovery signals from unseen glitches even though glitches play a key role in the evolution of a pulsar's spin frequency. We conclude that, at least over decadal time scales, the value of $n$ can be significantly larger than the canonical 3 and discuss the implications for the evolution of pulsars.
\end{abstract}

Key words: stars: neutron; pulsars: general; methods: data analysis;

\section{INTRODUCTION}

The spin-down of pulsars is one of the most prominent features of their rotation (Davies et al. 1969, Cole 1969). Considering the magnetic field of a neutron star in a vacuum to have dipolar configuration and assuming that the magnetic axis is misaligned with the rotation axis, it must radiate and lose energy, which contributes to the observed slow down (Gunn \& Ostriker 1969). Under the simplest assumption that magnetic dipole radiation is the only braking mechanism, we expect the spin-frequency $(v)$ and the spinfrequency derivative $(\dot{v})$ to follow, $\dot{v} \propto-v^{3}$. However, pulsars reside in dense plasma environments in which particles are accelerated up to very high energies. Such plasma outflows can result in radial deformation of pulsar's magnetic field lines, which could result in a large fraction of the angular mo-

^ E-mail: aparthas@mpifr-bonn.mpg.de mentum and energy loss in the form of particles (Manchester et al. 1985, Harding et al. 1999), and additionally contribute to the underlying magnetic braking (Spitkovsky 2006). The observations of quasi-periodic magnetospheric processes correlated with spin-down measurements (Kramer et al. 2006, Lyne et al. 2010), strengthens the argument that a pulsar wind plays a substantial role in the pulsar braking mechanism. Observations of glitches, sudden spin-up events, alter the normal spin-down of pulsars (Espinoza et al. 2011a). Glitches are thought to arise from the transfer of angular momentum from a more rapidly spinning neutron star superfluid component to the crust (Baym et al. 1969, Anderson \& Itoh 1975, Melatos \& Warszawski 2009). Coupling and decoupling between the crust and the superfluid core can decrease the moment of inertia with time, leading to further departures from the conventional spin-down due to magnetic dipole braking (Ho \& Andersson 2012). Pulsar braking is also thought to be dependent on the temporal evolution 
of the magnetic field strength $(B)$ and the inclination angle between the magnetic and the rotational axis $(\alpha)$ (Tauris \& Konar 2001, Young et al. 2010, Lyne et al. 2013, Johnston \& Karastergiou 2017). Finally, gravitational wave emission can also contribute to the energy losses in a pulsar. Thus, the supposition that a neutron star is a magnetic dipole rotating in a vacuum must be treated with caution.

The braking index $(n)$ is used to parameterize the spin evolution of pulsars and is defined by relating the spinfrequency and its derivative through a power law:

$\dot{v}=-K v^{\mathrm{n}}$.

$K$ depends on the moment of inertia of the neutron star and its magnetic moment. Although $K$ is often assumed to be constant in time, deviations from a constant magnetic moment can occur due to decay (or growth) of the magnetic field (Blandford et al. 1983) or changes in the dipole alignment (Candy \& Blair 1986) which makes $K$ time-dependent (Blandford \& Romani 1988, McKinney 2006). Neglecting these effects allows us to write the braking index as,

$n=\frac{v \ddot{v}}{\dot{v}^{2}}$,

where $\ddot{v}$ is the second derivative of the spin-frequency.

The characteristic age of the pulsar is usually written as $\tau_{c}=-v / 2 \dot{v}$ with the implicit assumption that $n=3$ and that the initial period of the pulsar is much less than its current period. However, given the variety of phenomena discussed above that can potentially contribute to pulsar braking, the characteristic age must be taken with caution (when interpreting it as a bona-fide age estimate of the pulsar).

There are challenges associated with measuring the long-term $\ddot{v}$. In most cases, the value arising from pure spin-down is dominated by other phenomena like timing noise or glitches. Timing noise is caused by stochastic rotational irregularities of the neutron star about a steady state (Groth 1975) and is seen in all classes of pulsars (Hobbs et al. 2004, Shannon \& Cordes 2010, Namkham et al. 2019, Parthasarathy et al. 2019). The cause of timing noise has been attributed to both neutron star interiors, arising from the cross-coupling between the neutron star crust and its superfluid core (Jones 1990), or from magnetospheric torque fluctuations (Cheng 1987). Failure to model timing noise leads to biased estimates of pulsar parameters (Coles et al. 2011). Developing upon previous Bayesian pulsar timing frameworks (van Haasteren \& Levin 2013, Lentati et al. 2014), Parthasarathy et al. (2019) (hereafter PSJ19) characterise the timing noise as a power-law process (see Equation 4) in a sample of 85 high $\dot{E}$, young radio pulsars and report the median red-noise amplitude, $\log _{10}\left(\frac{A_{\text {red }}}{y r^{3 / 2}}\right)$ to be $-10.4_{-1.7}^{+1.8}$ and the spectral index $(\beta)$ to be $-5.2_{-3.8}^{+3.0}$ and show that the strength of timing noise scales proportionally to $v^{1}|\dot{v}|^{-0.6 \pm 0.1}$. The modelling of timing noise simultaneously with the timing model will allow for unbiased measurements of pulsar parameters.

The presence of glitches, and in particular the glitch recovery, further complicates the measurement of $\ddot{v}$ and hence the braking index. Following a glitch event, a pulsar enters a recovery stage during which an increase in the spin-down rate and a relaxation towards the pre-glitch rotational state is observed over a timescale of days to years (Yu et al. 2013,
Fuentes et al. 2017). Both the spin-up event and the subsequent recovery stage are interpreted as the presence of a superfluid component in the inner crust and core of the star (Haskell \& Melatos 2015). Johnston \& Galloway (1999) and Alpar \& Baykal (2006) point out that a post-glitch relaxation process can dominate the spin-down evolution of a pulsar resulting in large $(n>3)$ values of braking indices.

Because of the difficulties outlined above, it has been common in the literature to only report values of $n$ when they are close to the canonical value of 3 . Lyne et al. (2015a) reported a braking index of 2.35(1) for the Crab pulsar (PSR J0534+2200) from 45 years of timing data. They note that the braking index value is close to 2.5 in the intervals between glitches. Similarly, the braking index and spin-down properties of the Vela pulsar are also well studied. While Shannon et al. (2016) determined the long-term value of $n$ to be $<8$, Espinoza et al. (2017) reported $n=1.7(2)$ using different techniques. Using inter-glitch models to obtain spin-down rates, Akbal et al. (2017) measured $n=2.8(1)$. It is important to note the reported long-term values of $n$ are smaller than the values reported for the linear regimes between glitches (where $n \sim 30$ ).

Braking index values ranging from $\sim 0.03$ for PSR B0540-69 (Marshall et al. 2016) to 3.15 for PSR J1640-4631 (Archibald et al. 2016 have been reported. In PSR J1513-5908, the braking index was measured in a series of papers (Kaspi et al. 1994, Livingstone et al. 2005, Livingstone \& Kaspi 2011) with the updated value being $2.832 \pm 0.003$. This pulsar has never been observed to glitch in $\sim 25$ years of observations. In contrast, PSR J0537-6910 has the highest glitch rate of any pulsar (Middleditch et al. 2006) with $\sim 45$ observed glitches in $\sim 14$ years of observations. Antonopoulou et al. (2018) reported that the spinevolution of this pulsar is characterised by a well-defined negative braking index of -1.22(4). Using the same dataset, Ferdman et al. (2018) and Andersson et al. (2018) reported that the trend in the interglitch behaviour leads to a larger value of $n \sim 7$.

In this paper, we measure $n$ for a sample of young pulsars using Bayesian inference. In Section 2, we report the measured values of $n$ for the 19 pulsars in our sample. In Section 3, we use simulated and historical data sets to test the robustness of the measured values. In Section 4 we introduce a glitch recovery model to test if unseen glitches before our data set affect the measurement of $n$. In Section 5, using Monte Carlo simulations we investigate the presence of exponential glitch recoveries on long timescales. In Section 6, we develop a parameterization for the detectability of braking index and discuss the implications of high values of $n$ and the evolution of pulsars with time.

\section{MEASURED BRAKING INDICES}

In PSJ19, we described the observations and the methodology involved in obtaining timing solutions for 85 young radio pulsars observed with the CSIRO 64-m Parkes radio telescope. A total of 19 pulsars had a preferred model which included $\ddot{v}$ as shown in Table 3 of PSJ19. Here we reproduce the values of $\log _{10}\left(A_{\text {red }}\right), \beta, v, \dot{v}, \ddot{v}, n$ and the preferred model for these 19 pulsars in Table 1 . In addition we place upper limits on the remaining pulsars in the sample. For clarity, 
we redefine the various models listed in Table 1 below. In each timing model, we fitted for the pulsar position, spin $(v)$ and spin-down parameter $(\dot{v})$ in addition to

- a power-law timing noise and $\ddot{v}(\mathrm{PL}+\mathrm{F} 2)$,

- a power-law, $\ddot{v}$ and low-frequency components longer than the data set $(\mathrm{PL}+\mathrm{F} 2+\mathrm{LFC})$,

- a power-law, $\ddot{v}$ and proper motion $(\mathrm{PL}+\mathrm{F} 2+\mathrm{PM})$,

- a cut-off power law and a $\ddot{v}(\mathrm{CPL}+\mathrm{F} 2)$.

Figure 1 shows the derived values of $n$ (Equation 2) and the $97.5 \%$ upper limits.

Of the 19 braking index measurements, four of them have $n<10$, eleven have $10<n<100$, two of them are $>$ 100 and two of them are negative. Only for PSR J1513-5908 is the value close to $n=3$ as expected from a simple model of a magnetic dipole in vacuum. Two questions thus arise. The first is whether the measured values of $n$ are robust to the passage of time and the second is whether or not unseen glitches affect the measured values of $n$ ? We answer these questions in the next three sections.

\section{ROBUSTNESS OF THE BRAKING INDEX MEASUREMENTS}

\subsection{Comparisons with simulated data sets.}

We first test the efficacy of TEMPONEST (Lentati et al. 2014) in this context, by simulating timing residuals for the 19 pulsars discussed in this paper with various induced signatures as per the preferred model and check the consistency with which we recover the input parameters. The simulated timing residuals are generated using LIBSTEMPO ${ }^{1}$ with data spans similar to the actual observations. The times of arrival (ToAs) are considered to be a combination of deterministic and stochastic components. White noise components are modelled by adjusting the uncertainty on a ToA to be,

$\sigma^{2}=F \sigma_{\mathrm{r}}^{2}+\sigma_{\mathrm{Q}}^{2}$,

where the parameter $F$, referred to as the EFAC, accounts for instrumental distortions and ${\sigma_{\mathrm{r}}}^{2}$ is the formal uncertainty obtained from ToA fitting. An $\operatorname{EQUAD}\left(\sigma_{\mathrm{Q}}^{2}\right)$ is introduced to model time independent noise per observing system. The timing noise is modelled to be a power-law described by a red-noise amplitude $\left(A_{\text {red }}\right)$ and spectral index $(\beta)$,

$P_{\mathrm{r}}(f)=\frac{A_{\mathrm{red}}^{2}}{12 \pi^{2}}\left(\frac{f}{f_{\mathrm{yr}}}\right)^{-\beta}$,

where $f_{y r}$ is a reference frequency of 1 cycle per year and $A_{\text {red }}$ is in units of $\mathrm{yr}^{3 / 2}$. A model with a cut-off power law was favoured for PSR J1830-1059 which was simulated following the power spectrum,

$P_{\mathrm{r}, \mathrm{CF}}(f)=\frac{A\left(f_{\mathrm{c}} / f_{\mathrm{yr}}\right)^{-\beta}}{\left[1+\left(f / f_{\mathrm{c}}\right)^{-\beta / 2}\right]^{2}}$,

where $f_{\mathrm{c}}$ is the corner frequency and $A$ is $\left(A_{\text {red }}^{2} / 12 \pi^{2}\right)$.

To maintain consistency in the simulations, a model with low-frequency components (LFC) with timescales

1 https://github.com/vallis/libstempo longer than the data set, were simulated for PSRs J16434505, J1815-1738 and J1824-1945 and a model with a proper motion signature was simulated for PSR J1809-1917. Finally, the simulated timing residuals were modified to include a braking index signature.

We use the Bayesian pulsar timing package, TEMPONEST to search for the stochastic parameters and the braking index value in the simulated data sets. The prior ranges on the stochastic parameters, corner frequency, low-frequency components, $\ddot{v}$ and the proper motion are reported in Table 2. Our measurements of the stochastic, braking index and other induced parameters (proper motion, corner frequency and LFC) are consistent between the observed and simulated data sets for all 19 pulsars. Figures 2 and 3 show the consistency in the measured values of red noise amplitudes, spectral indices, and the derived braking index values from the real and simulated data sets for a sample of six pulsars. The shaded regions in the plots represent $50 \%$ and $95 \%$ confidence intervals.

\subsection{Using legacy data sets.}

We obtained historical data for 9 of the 19 pulsars and prepended these data to those used in PSJ19. The historical data is also at $20-\mathrm{cm}$ wavelengths observed with the $64-\mathrm{m}$ CSIRO Parkes radio telescope. The data set used in PSJ19 is hereafter called the PSJ19-data set and the data set that includes the historical data is called the legacy data set. Figure 4 shows the timing residuals for these nine pulsars with the added data.

Figure 5 shows the posterior distributions for six pulsars that have consistent values (overlapping confidence intervals) of $\ddot{v}$, braking index and the timing noise parameters using the legacy data set. A model with power-law timing noise and $\ddot{v}(\mathrm{PL}+\mathrm{F} 2)$ was still favoured for three pulsars and PL+F2+LFC for PSR J1824-1945. For PSR J1412-6145, we fitted for the glitch parameters as reported in Yu et al. (2013) while simultaneously modelling the stochastic and braking index parameters. The preferred model in this case is one with the glitch parameters (PL+F2+GL). For PSR J18330827, we measure a proper motion signal with the legacy data set, with the proper motion in right ascension $\left(\mu_{\alpha}\right)$ being $-37 \pm 7 \mathrm{mas} \mathrm{yr}^{-1}$, in declination $\left(\mu_{\delta}\right)$ being $3 \pm 26{\text { mas } \mathrm{yr}^{-1}}^{-1}$

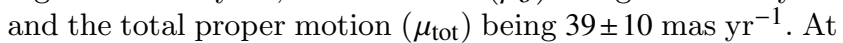
a distance of $4.38 \mathrm{kpc}$ (from DM estimates), this amounts to a high transverse velocity of $800 \pm 200 \mathrm{kms}^{-1}$. The preferred timing model with the legacy data set is $\mathrm{PL}+\mathrm{F} 2+\mathrm{PM}$ with a $\log$ Bayes factor of 21 . It must be noted that the measured braking index remains unchanged within uncertainties. PSRs J1513-5908, J1632-4818 and J1715-3903 exhibited clear inconsistencies in either the $\ddot{v}$ or the timing noise parameters after adding archival data. These are further investigated below on a case-by-case basis.

PSR J1513-5908: This pulsar has not been observed to glitch. With 28.4 years of radio timing data from 1982 June to 2010 November, Livingstone \& Kaspi (2011) reported a braking index value of $2.832 \pm 0.003$. They also reported a detection of the third derivative of the spinfrequency $(\dddot{v})$ to be $-0.9139(2) \times 10^{-31} \mathrm{~s}^{-4}$.

We notice that a model with a $\dddot{v}$ is preferred over the $\mathrm{PL}+\mathrm{F} 2$ model in the legacy data and including it as part of the timing model results in consistent measurements of 
Table 1. The timing noise parameters $A_{\text {red }}, \beta$ (as defined in equation 4 ), the measured spin parameters $(v, \dot{v}, \ddot{v})$ along with the braking index $(n)$ values and the preferred model (as described in Section 2) for 19 pulsars.

\begin{tabular}{|c|c|c|c|c|c|c|c|}
\hline PSR & $\begin{array}{r}\log _{10}\left(A_{\mathrm{red}}\right) \\
\left(\mathrm{yr}^{3 / 2}\right)\end{array}$ & $\beta$ & $\begin{array}{r}v \\
\left(s^{-1}\right)\end{array}$ & $\begin{array}{r}\dot{v} \\
\left(10^{-14} s^{-2}\right)\end{array}$ & $\begin{array}{r}\ddot{v} \\
\left(10^{-23} s^{-3}\right)\end{array}$ & $n$ & Preferred Model \\
\hline J0857-4424 & $-11.3_{-0.6}^{+1.2}$ & $-9.1_{-1.6}^{+3.8}$ & $3.0601045423(4)$ & $-19.6145(10)$ & $3.63(16)$ & $2890(30)$ & $\mathrm{PL}+\mathrm{F} 2$ \\
\hline J0954-5430 & $-10.4_{-0.3}^{+0.6}$ & $-4.4_{-0.8}^{+2.1}$ & $2.11483307064(18)$ & $-19.6358(5)$ & $0.032(8)$ & $18(9)$ & $\mathrm{PL}+\mathrm{F} 2$ \\
\hline $\mathrm{J} 1412-6145$ & $-10.7_{-0.6}^{+1.1}$ & $-7.9_{-1.6}^{+3.6}$ & $3.1720007909(13)$ & $-99.643(4)$ & $0.62(4)$ & $20(3)$ & $\mathrm{PL}+\mathrm{F} 2$ \\
\hline J1509-5850 & $-11.1_{-2.1}^{+3.1}$ & $-5.1_{-2.9}^{+8.6}$ & $11.2454488757(7)$ & $-115.9175(16)$ & $0.12(16)$ & $11(3)$ & $\mathrm{PL}+\mathrm{F} 2$ \\
\hline J1513-5908 & $-9.7_{-0.2}^{+0.4}$ & $-5.7_{-0.6}^{+1.3}$ & $6.59709182778(19)$ & $-6653.10558(27)$ & $189.6(2)$ & $2.82(6)$ & $\mathrm{PL}+\mathrm{F} 2$ \\
\hline $\mathrm{J} 1524-5706$ & $-10.2_{-0.7}^{+1.0}$ & $-3.6_{-1.3}^{+3.6}$ & $0.89591729463(9)$ & $-28.60366(2)$ & $0.038(2)$ & $4.2(7)$ & $\mathrm{PL}+\mathrm{F} 2$ \\
\hline $\mathrm{J} 1531-5610$ & $-11.8_{-0.6}^{+1.3}$ & $-8.5_{-1.6}^{+3.4}$ & $11.8756292823(4)$ & $-194.5360(14)$ & $1.37(2)$ & $43(1)$ & $\mathrm{PL}+\mathrm{F} 2$ \\
\hline $\mathrm{J} 1632-4818$ & $-9.6_{-0.5}^{+0.8}$ & $-5.0_{-1.1}^{+2.7}$ & $1.2289964712(14)$ & $-98.0730(3)$ & $0.48(4)$ & $6(1)$ & $\mathrm{PL}+\mathrm{F} 2$ \\
\hline $\mathrm{J} 1637-4642$ & $-9.7_{-0.3}^{+0.6}$ & $-4.9_{-0.9}^{+2.2}$ & $6.491542203(4)$ & $-249.892(10)$ & $3.2(15)$ & $34(3)$ & $\mathrm{PL}+\mathrm{F} 2$ \\
\hline J1643-4505 & $-10.1_{-0.3}^{+0.5}$ & $-2.3_{-0.4}^{+1.0}$ & $4.212470392(4)$ & $-56.473(10)$ & $0.11(2)$ & $15(6)$ & $\mathrm{PL}+\mathrm{F} 2+\mathrm{LFC}$ \\
\hline $\mathrm{J} 1648-4611$ & $-10.4_{-0.5}^{+0.8}$ & $-6.3_{-0.9}^{+2.3}$ & $6.0621606076(2)$ & $-87.220(5)$ & $0.44(8)$ & $40(10)$ & $\mathrm{PL}+\mathrm{F} 2$ \\
\hline J1715-3903 & $-9.2_{-0.1}^{+0.2}$ & $-3.8_{-0.6}^{+1.3}$ & $3.5907423095(9)$ & $-48.2784(13)$ & $0.4(11)$ & $70(40)$ & $\mathrm{PL}+\mathrm{F} 2$ \\
\hline J1738-2955 & $-9.6_{-0.2}^{+0.5}$ & $-5.8_{-1.0}^{+2.4}$ & $2.2551713364(2)$ & $-41.7146(12)$ & $-0.51(16)$ & $-70(40)$ & $\mathrm{PL}+\mathrm{F} 2$ \\
\hline $\mathrm{J} 1806-2125$ & $-9.1_{-0.1}^{+0.3}$ & $-6.6_{-0.7}^{+1.6}$ & $2.075444041(15)$ & $-50.821(2)$ & $1.1(4)$ & $90(60)$ & $\mathrm{PL}+\mathrm{F} 2$ \\
\hline J1809-1917 & $-11.7_{-0.6}^{+1.1}$ & $-9.0_{-1.4}^{+3.5}$ & $12.0838226201(8)$ & $-372.7882(19)$ & $2.70(3)$ & $23.5(6)$ & $\mathrm{PL}+\mathrm{PM}+\mathrm{F} 2$ \\
\hline J1815-1738 & $-11.8_{-1.5}^{+3.1}$ & $-4.5_{-1.4}^{+3.1}$ & $5.03887545888(10)$ & $-197.4552(11)$ & $0.73(8)$ & $9(3)$ & $\mathrm{PL}+\mathrm{F} 2+\mathrm{LFC}$ \\
\hline J1824-1945 & $-10.9_{-0.1}^{+0.3}$ & $-3.4_{-0.3}^{+0.6}$ & $5.281575552287(3)$ & $-14.6048(5)$ & $0.05(2)$ & $120(20)$ & $\mathrm{PL}+\mathrm{F} 2+\mathrm{LFC}$ \\
\hline J1830-1059 & $-8.5_{-0.1}^{+0.3}$ & $-13.6_{-2.8}^{+6.2}$ & $2.4686900068(5)$ & $-36.5201(10)$ & $0.167(19)$ & $31(7)$ & $\mathrm{CPL}+\mathrm{F} 2$ \\
\hline J1833-0827 & $-10.2_{-0.1}^{+0.2}$ & $-2.8_{-0.6}^{+1.2}$ & $11.7249580817(4)$ & $-126.1600(8)$ & $-0.198(13)$ & $-15(2)$ & $\mathrm{PL}+\mathrm{F} 2$ \\
\hline
\end{tabular}

Table 2. Prior ranges for the various stochastic and deterministic parameters used in the timing models. $\Delta_{\text {param }}$ is the uncertainty on the marginalized timing model parameters from the initial TEMPO2 fitting.

\begin{tabular}{lrr}
\hline \hline Parameter & Prior range & Type \\
& & \\
\hline Red noise amplitude $\left(A_{\text {red }}\right)$ & $(-20,-5)$ & Log-uniform \\
Red noise slope $(\beta)$ & $(0,20)$ & Log-uniform \\
EFAC & $(-1,1.2)$ & Log-uniform \\
EQUAD & $(-10,-3)$ & Log-uniform \\
Corner frequency $\left(f_{\mathcal{c}}\right)$ & $\left(0.01 / T_{\text {span }}, 10 / T_{\text {span }}\right)$ & Log-uniform \\
Low frequency cut-off $($ LFC $)$ & $(-1,0)$ & Log-uniform \\
$\ddot{\nu}$ & $\pm 10000 \times \Delta_{\text {param }}$ & Uniform \\
Proper motion & \pm 1000 mas $/$ yr & Uniform \\
\hline
\end{tabular}

$\ddot{v}$ and the timing noise parameters. An expression for $\dddot{v}$ can be derived by taking two derivatives of equation 1 and is written as,

$\dddot{v}=\frac{n(2 n-1) \dot{v}^{3}}{v^{2}}$.

The validity of the spin-down law can then be tested by calculating the expected second braking index $\left(m_{0}\right)$, which is written as,

$m_{0} \equiv n(2 n-1)$,

and if equation 1 accurately describes the spin-down of the pulsar, then $m_{0}=m$, where $m$ is,

$m=\frac{v^{2} \dddot{v}}{\dot{v}^{3}}$.

A value of $m$ can be measured from higher order frequency derivatives of a pulsar, which is only possible in the youngest pulsars that have large spin-down and minimal timing noise. We measure the value of $m$ to be $14.4 \pm 2.2$ and the computed value of $m_{0}$ to be $13.23 \pm 0.03$, which are in agreement with each other within uncertainties. In Figure 6 we show our measurements of $\ddot{v}, \dddot{v}$ (F3), $n, m_{0}, m$ and the timing noise parameters which are consistent with the measurements reported in Livingstone et al. (2005) within 95\% confidence limits. Our measurements of timing noise and the absence of glitches in this pulsar are in contrast to the suggestions made by Hobbs et al. (2010) that timing noise in pulsars with characteristic ages less than $10^{5} \mathrm{yr}$ is due to unmodelled glitch recovery.

PSR J1632-4818: Using TEMPONEST, we searched for glitches in the legacy data set and found evidence for the presence of a glitch at MJD 53962 10 days and with a glitch 


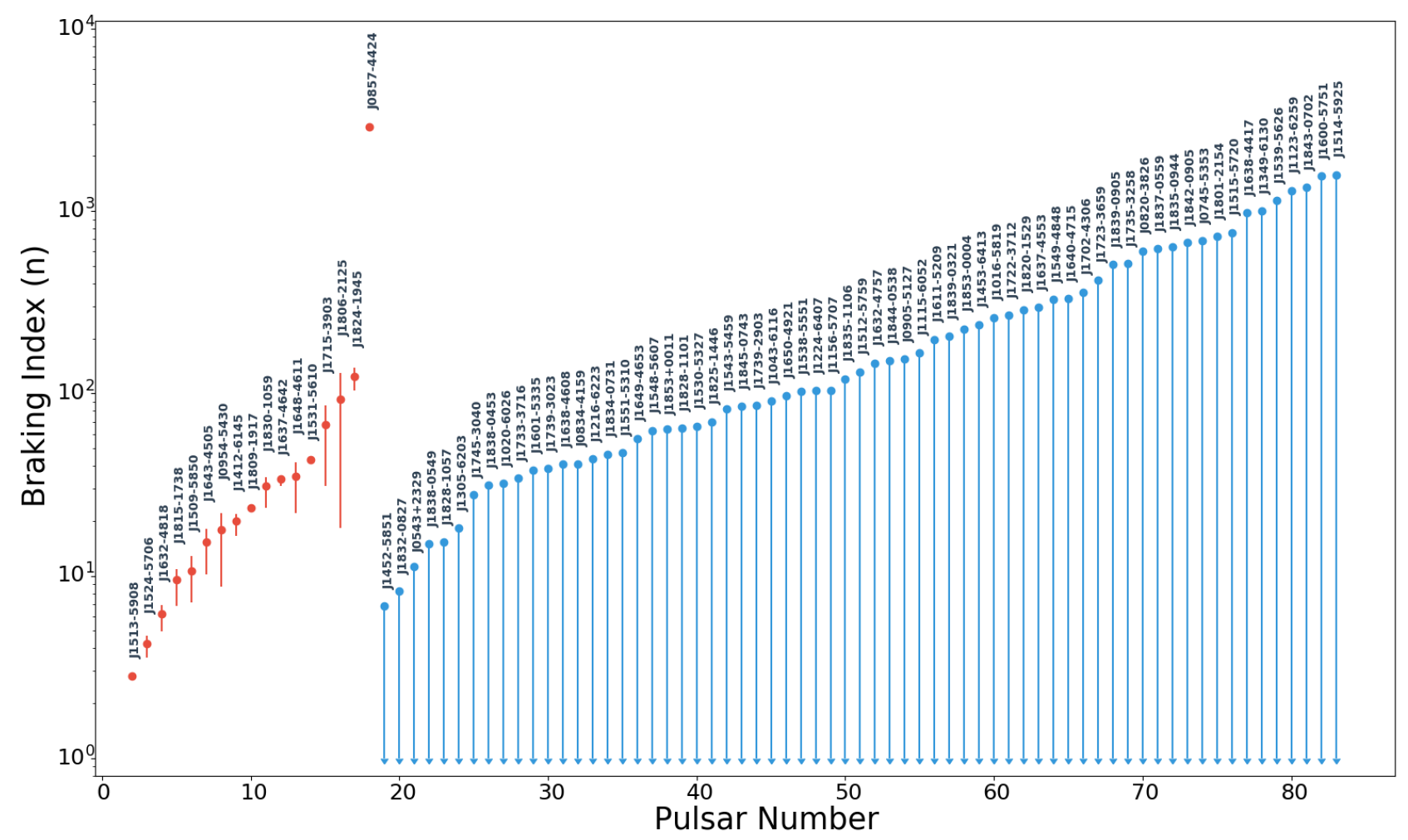

Figure 1. Braking index detections (red points) and upper limits (blue points) on our sample of 85 pulsars, excluding the 2 pulsars with negative braking indices. The error bars are $97.5 \%$ and $2.5 \%$ limits on the detections and $97.5 \%$ upper limits on the non-detections.

amplitude of $(1.1 \pm 0.2) \times 10^{-8} \mathrm{~Hz}$. The detection was also supported by the fact that the log Bayes factor for the glitchsearch model was 17, which is much higher than our Bayes factor threshold of 5 (used in PSJ19). Figure 7 shows the posterior distributions of timing noise, $\ddot{v}$ and braking index measurements for the PSJ19 and legacy data sets, before and after accounting for the glitch.

PSR J1715-3903: Similar to PSR J1632-4818, we found evidence for the presence of a glitch at MJD $57724 \pm 2$ days in the PSJ19 data set, with a glitch amplitude of $(1.9 \pm 0.1) \times 10^{-8} \mathrm{~Hz}$ and the glitch model is strongly preferred over a model without the glitch with a Bayes factor of 35 . Figure 8 shows the measured posterior distributions of $\ddot{v}$, braking index and the timing noise parameters for both the data sets before and after modelling for the glitch. It is evident from Figure 8 that the uncertainties on the measurement of the braking index have improved after accounting for the glitch.

\subsection{Summary}

In Table 3, we report the time-span of the legacy data sets along with the updated braking index values using the updated timing models for PSR J1412-6145, J1513-5908, J1632-4818 and J1715-3903. There are three important results from this section. First, we showed through simulations that TEMPONEST is capable of recovering braking indices in the presence of strong timing noise. Secondly, we demonstrated that TEMPONEST is capable of detecting glitches within the time span of the datasets. Thirdly, we demonstrated that increasing the overall data span maintains the value of $n$ even in the presence of glitch activity.

\section{GLITCH RECOVERY}

Here we investigate whether unseen glitches in the past affect the measured value of $n$. Of crucial importance is not the glitch size itself but rather the recovery from the glitch. Generally the form of the recovery is taken to be an exponential which asymptotes towards a frequency increment parameter, $v_{\mathrm{GLD}}$, with a glitch decay time of $\tau_{\mathrm{GLD}}$. Typically, glitch recovery is expressed as $Q \equiv v_{\mathrm{GLD}} / v_{\mathrm{g}}$, where $v_{\mathrm{g}}$ is the total increment in the pulsar spin-frequency due to the glitch, expressed as $v_{\mathrm{GLD}}+v_{\mathrm{p}}$. Here, $v_{\mathrm{p}}$ is the permanent increment in the pulse frequency. The exponential form of the recovery implies that the epoch of the glitch is not important and since we are interested in glitches before our data set, the $v_{\mathrm{p}}$ is set to zero. In what follows therefore, the glitch is assumed to occur one day prior to the start of our data sets. We also keep in mind that the presence of $\ddot{v}$ (and hence a braking index) manifests itself as a cubic in the timing residuals.

We can imagine several cases of interest. First, it is clear that very small values of $v_{\mathrm{GLD}}$ and/or small decay times will have little effect on our ability to measure $\ddot{v}$. For large decay times (longer than our dataset) the exponential looks linear and will be absorbed into the fits of $v$ and $\dot{v}$ and will hence not affect $\ddot{v}$. However, for decay times comparable to our data span (i.e. from 1000 days to 5000 days) the exponential 

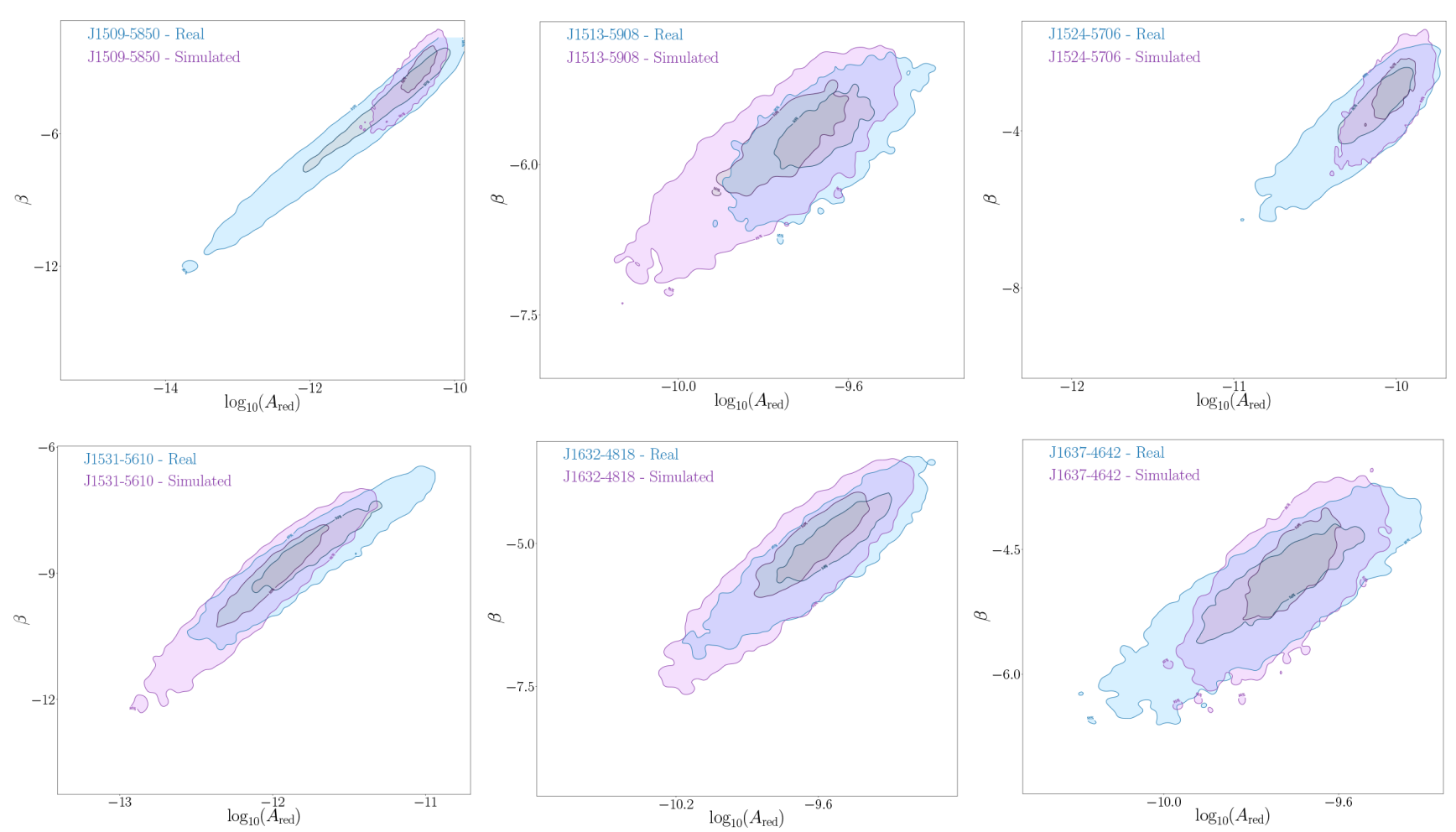

Figure 2. Posterior distributions of the red noise amplitudes and spectral indices for a sample of six pulsars. The blue posteriors represent real data, while the purple posteriors represent simulated data.

Table 3. Braking index measurements for nine pulsars before and after increasing the time span of the data as measured from the preferred timing models.

\begin{tabular}{|c|c|c|c|c|c|c|c|}
\hline PSR & $\begin{array}{r}\text { Old timespan } \\
(\mathrm{yr})\end{array}$ & New MJD Range & $\begin{array}{r}\text { New timespan } \\
(\mathrm{yr})\end{array}$ & Old braking index & New braking index & Old timing model & New timing model \\
\hline J0954-5430 & 10 & $50849-57824$ & 19 & $18(9)$ & $15(3)$ & $\mathrm{PL}+\mathrm{F} 2$ & $\mathrm{PL}+\mathrm{F} 2$ \\
\hline J1412-6145 & 10 & $51782-58012$ & 16.9 & $20(3)$ & $19(2)$ & $\mathrm{PL}+\mathrm{F} 2$ & $\mathrm{PL}+\mathrm{F} 2+\mathrm{GL}$ \\
\hline J1509-5850 & 10 & $51221-58012$ & 18.5 & $11(3)$ & $11.2(5)$ & $\mathrm{PL}+\mathrm{F} 2$ & $\mathrm{PL}+\mathrm{F} 2$ \\
\hline J1513-5908 & 11.6 & $48732-58012$ & 25 & $2.826(3)$ & $2.834(3)$ & $\mathrm{PL}+\mathrm{F} 2$ & $\mathrm{PL}+\mathrm{F} 2+\mathrm{F} 3$ \\
\hline J1524-5706 & 10 & $51212-58012$ & 18.6 & $4.3(7)$ & $4.2(1)$ & $\mathrm{PL}+\mathrm{F} 2$ & $\mathrm{PL}+\mathrm{F} 2$ \\
\hline J1632-4818 & 10 & $51782-58012$ & 14 & $6.2(7)$ & $5.9(5)$ & $\mathrm{PL}+\mathrm{F} 2$ & $\mathrm{PL}+\mathrm{F} 2+\mathrm{GL}$ \\
\hline J1715-3903 & 10 & $51878-58012$ & 14 & $70(40)$ & $68(10)$ & $\mathrm{PL}+\mathrm{F} 2$ & $\mathrm{PL}+\mathrm{F} 2+\mathrm{GL}$ \\
\hline J1824-1945 & 11.6 & $51844-58012$ & 18 & $120(20)$ & $88(15)$ & $\mathrm{PL}+\mathrm{F} 2+\mathrm{LFC}$ & $\mathrm{PL}+\mathrm{F} 2+\mathrm{LFC}$ \\
\hline J1833-0827 & 10 & $51782-58012$ & 16.6 & $-15(2)$ & $-14.2(6)$ & $\mathrm{PL}+\mathrm{F} 2$ & $\mathrm{PL}+\mathrm{F} 2+\mathrm{PM}$ \\
\hline
\end{tabular}

mimics a (partial) cubic, and can therefore masquerade as a $\ddot{v}$ term. This will always serve to increase $\ddot{v}$ and hence also increase the value of $n$. Larger values of $v_{\mathrm{GLD}}$ will lead to larger values of $n$. In general, the induced value of $n$ is largely independent of the other pulsar parameters; it depends only on $v_{\mathrm{GLD}}$ and $\tau_{\mathrm{GLD}}$. However, the presence of timing noise complicates such analysis.

We assume the presence of an unseen glitch before the data set for each of our 19 pulsars and search for evidence of glitch recovery signals spanning a $v_{\mathrm{GLD}}$ range of $10^{-14}$ to $10^{-3} \mathrm{~Hz}$ and a $\tau_{\mathrm{GLD}}$ range of 1 to 10,000 days. We use a log-uniform prior for the glitch decay parameters. The prior ranges for the timing noise parameters and $\ddot{v}$ are as reported in Table 2. We then fitted for three different timing models and compared their log-evidences. These are:
- the timing model as reported in Tables 1 and 3,

- the timing model with a glitch recovery signal (TimingModel+GLR)

- the timing model, without a cubic polynomial (F2), but including a glitch recovery signal (TimingModel-F2+GLR)

For PSR J0954-5430, the three models that we compare are $\mathrm{PL}+\mathrm{F} 2, \mathrm{PL}+\mathrm{F} 2+\mathrm{GLR}$ and $\mathrm{PL}+\mathrm{GLR}$, since the timing model as reported in Table 1 is PL+F2. However, for PSR J1412-6145, since the updated timing model reported in Table 3 is $\mathrm{PL}+\mathrm{F} 2+\mathrm{GL}$, the three models to compare are $\mathrm{PL}+\mathrm{F} 2+\mathrm{GL}, \mathrm{PL}+\mathrm{F} 2+\mathrm{GL}+\mathrm{GLR}$ and PL+GL+GLR (without the F2).

Table 4 reports the log-evidences for the three timing models for 19 pulsars in our sample. The preferred model for each pulsar is highlighted. Two things are apparent; 

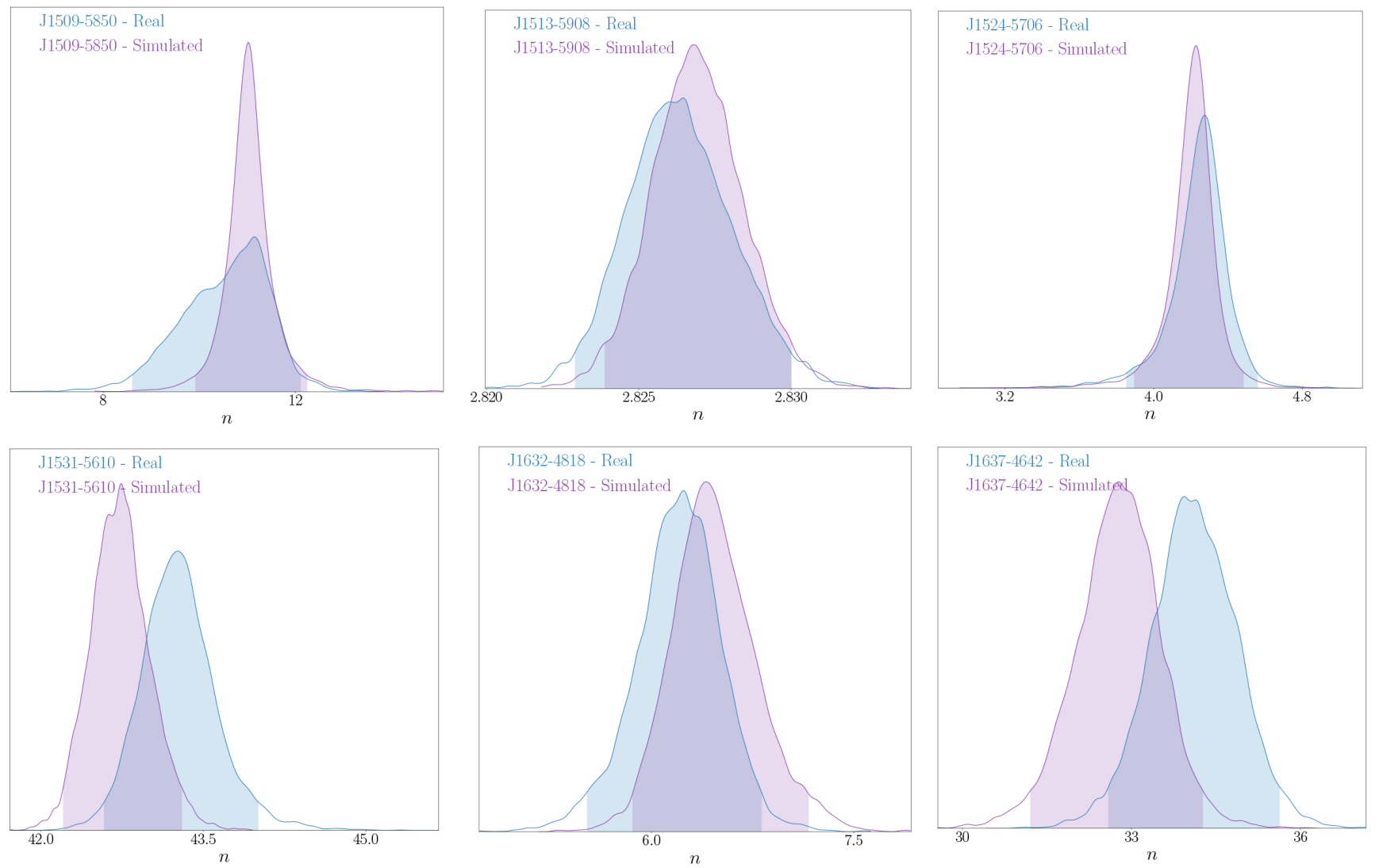

Figure 3. Posterior distributions of the braking index measurements for a sample of six pulsars. The blue posteriors represent real data, while the purple posteriors represent simulated data.

first that there is much stronger evidence for a cubic term than for an exponential. Secondly, that although TimingModel+GLR and TimingModel have similar log-evidences, the simpler model would always be preferred. This is following the Occam principle, which states that all other things being equal, a simpler model is more likely than a complicated one. Taken together, this shows that there is little evidence for an exponential signal in the data. It is also important to note that the Bayesian inference framework enables us to identify cases when we cannot distinguish between models. In this case, distinguishing between a braking index and a glitch recovery signal is important while interpreting the measurements.

Finally we note that PSRs J1531-5610, J1806-2125, J1809-1917 and J1833-0827 have glitches detected before our data span. The glitch sizes are a few $\times 10^{-5} \mathrm{~Hz}$ as reported in Yu et al. (2013) and Espinoza et al. (2011a) and the difference between the MJD of the first ToA in our data set and the glitch epochs are 2500, 2300, 1000 and 4200 days respectively. Yu et al. (2013) also measure glitch recovery times of a few hundred days for 3 of these pulsars. If correct, then no evidence of the glitch would remain in the residuals of our data as is confirmed by Table 4 .
Table 4. Comparison of log-evidence values for models with glitch recovery and braking index for 19 pulsars. The preferred model for each pulsar is highlighted.

\begin{tabular}{lrrr}
\hline \hline PSR & $\begin{array}{r}\text { TimingModel+GLR } \\
\text { (with F2 \& GLR) }\end{array}$ & $\begin{array}{r}\text { TimingModel } \\
\text { (with only F2) }\end{array}$ & $\begin{array}{r}\text { TimingModel-F2+GLR } \\
\text { (with only GLR) }\end{array}$ \\
\hline J0857-4424 & 1144 & $\mathbf{1 1 5 0}$ & 807 \\
J0954-5430 & 1357 & $\mathbf{1 3 5 8}$ & 934 \\
J1412-6145 & 1184 & $\mathbf{1 1 9 7}$ & 1165 \\
J1509-5850 & 1593 & $\mathbf{1 5 9 4}$ & 1565 \\
J1513-5908 & 2961 & $\mathbf{2 9 6 2}$ & 2452 \\
J1524-5706 & 1156 & $\mathbf{1 1 5 7}$ & 721 \\
J1531-5610 & 1082 & $\mathbf{1 0 8 3}$ & 1056 \\
J1632-4818 & 717 & $\mathbf{7 2 5}$ & 460 \\
J1637-4642 & 727 & $\mathbf{7 2 8}$ & 684 \\
J1643-4505 & 705 & $\mathbf{7 0 6}$ & 698 \\
J1648-4611 & 739 & $\mathbf{7 4 6}$ & 707 \\
J1715-3903 & 893 & $\mathbf{9 0 1}$ & 725 \\
J1738-2955 & 510 & $\mathbf{5 1 1}$ & 481 \\
J1806-2125 & 572 & $\mathbf{5 7 2}$ & 528 \\
J1809-1917 & 943 & $\mathbf{9 4 6}$ & 774 \\
J1815-1738 & 517 & $\mathbf{5 1 8}$ & 510 \\
J1824-1945 & 1546 & $\mathbf{1 5 4 8}$ & 986 \\
J1830-1059 & 958 & $\mathbf{9 6 0}$ & 943 \\
J1833-0827 & 1345 & $\mathbf{1 3 4 6}$ & 617 \\
\hline
\end{tabular}

\section{GLITCH SIMULATIONS}

We have shown in the above section that there is no (Bayesian) evidence for the presence of an exponential glitch recovery signal in our data, even in those pulsars known to have glitched prior to our data set. However, we also noted that a long decay time (> 1000 days) could induce a positive 


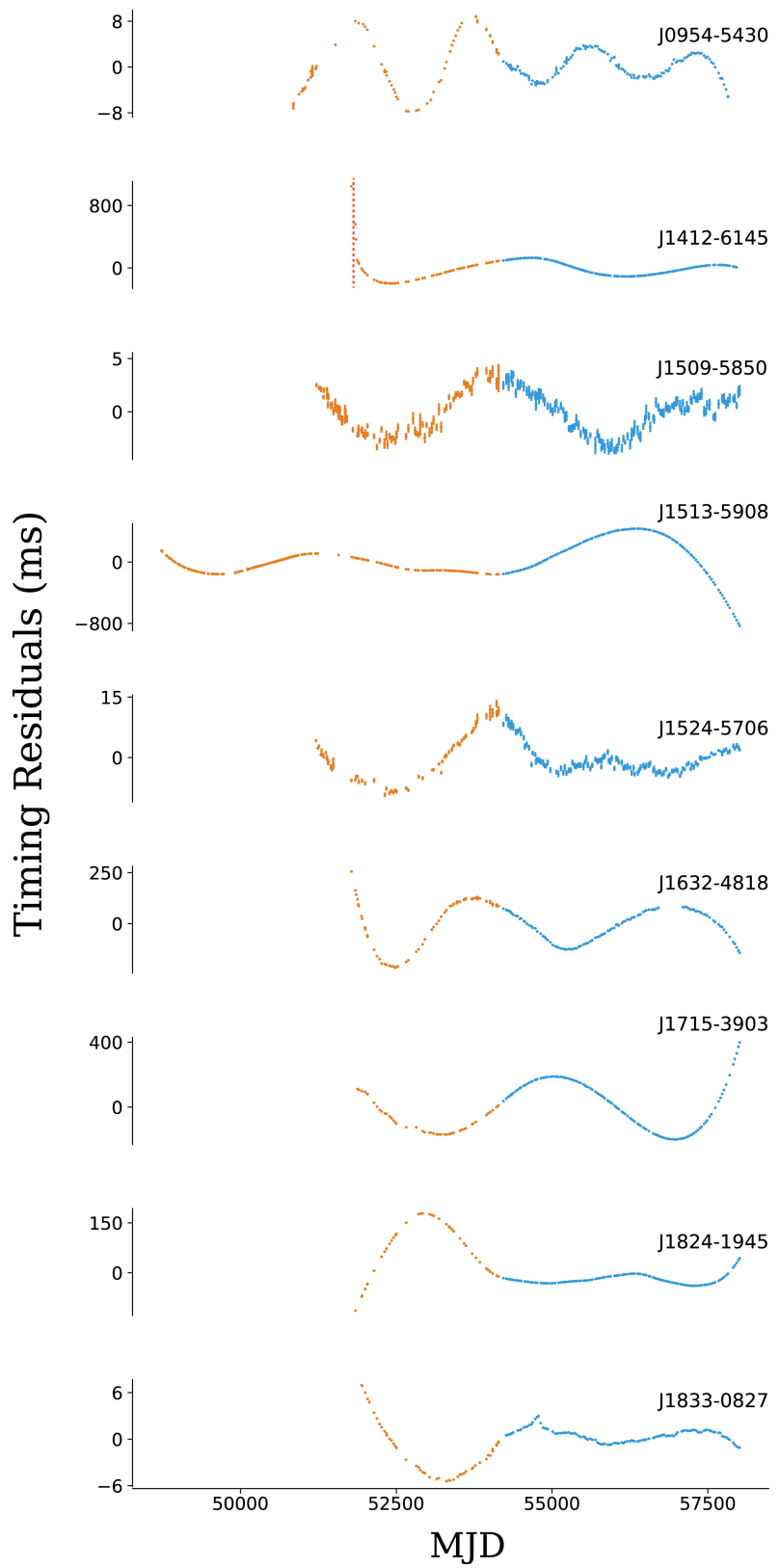

Figure 4. Phase connected timing residuals for nine pulsars with added archival data sets (orange) are shown here before subtracting the timing noise model. The vertical dashed line in PSR J1412-6145 shows the epoch of the glitch reported in Yu et al. (2013). For PSRs J1632-4818 and J1715-3903, the new glitches described in Section 3.2 are modelled in these timing residual plots.

$n$ in the residuals. In this section, we therefore consider the possibility of long glitch recovering times contaminating our data.

Using the TEMPO2 package, we measure the induced $\ddot{v}$ as determined when fitting for F2 for different simulated data sets with a typical data span of $15 \mathrm{yr}$, and for 1000 days $<\tau_{\mathrm{GLD}}<10000$ days. Using these measurements, we empirically determine the link between $v_{\mathrm{GLD}}, \tau_{\mathrm{GLD}}$ and the induced $\ddot{v}$ as

$\ddot{v}=10^{-14} \frac{v_{\mathrm{GLD}}}{\tau_{\mathrm{GLD}}} s^{-3}$

with $v_{\mathrm{GLD}}$ in $\mathrm{Hz}$ and $\tau_{\mathrm{GLD}}$ in days. For a given pulsar this translates into an induced $n$ via Equation 2. Second, for each pulsar we compute the glitch rate and typical glitch size. For this we use the metric for the glitch rate given by Fuentes et al. (2017) along with the parameterisation of large glitch sizes given in the same paper. As a check on the metric, we use Poisson statistics and determine that we expect $\sim 3.2$ pulsars in our sample to have glitched in the 15 year observing span of the data. We have shown above that indeed 3 pulsars have undergone glitches in this time span, in good agreement with the predictions of Fuentes et al. (2017).

We then perform a Monte Carlo simulation in the following way:

- For each pulsar, we determine the glitch rate, and then using Poisson statistics randomly select a date in the past for the glitch to have occurred.

- Draw a glitch size from the distribution of large glitches given in Fuentes et al. (2017).

- For a given $\tau_{\mathrm{GLD}}$, extrapolate forwards in time to the start of our data set and then compute the effective glitch size at that date assuming an exponential recovery.

- Compute the induced $\ddot{v}$ and hence $n$ from Equation 9 above.

- If the induced $n$ is greater than the upper limit for a given pulsar from Table 1 then count this as a detection.

The output of the simulation shows that the most likely 'detections' of $n$ are, unsurprisingly, in pulsars with a high glitch rate and/or for which the upper limit on $n$ is small. For example, PSR J1809-1917 has the highest detection probability of 0.98 with a median induced $n$ of 21 followed by PSR J1531-5610 with a detection probability of 0.86 and a median induced $n$ of 37.2. Both these pulsars feature in Table 1. The question now arises as to how the addition of timing noise affects these results and whether TEMPONEST can detect long timescale glitch recovery signals.

We consider the cases of PSR J1809-1917 and J15315610. We simulate a $10 \mathrm{yr}$ data set with an induced $v_{\mathrm{GLD}}$ of $4 \times 10^{-6} \mathrm{~Hz}$, a $\tau_{\mathrm{GLD}}$ of 5000 days and the glitch epoch set one day before the first data set. A power-law timing noise signal is induced with $A_{\text {red }}$ and $\beta$ as in Table 1 . We set $\ddot{v}=0$. Fitting for the three different models; PL $+\mathrm{F} 2$, $\mathrm{PL}+\mathrm{GLR}$ and PL+F2+GLR using TEMPONEST, we find that in both the cases, the PL+GLR model is preferred over the PL+F2+GLR model (with Bayes factors of 12 and 7 respectively), while the PL+F2 model is strongly disfavoured (with Bayes factors of -200).

These simulations together with the results from Table 4 have shown that:

- the braking indices are robust to the addition of legacy data, which is crucial in indicating a lack of exponential signal in the PSJ19 data,

- the model with $\ddot{v}$ is preferred over an exponential glitch recovery model for all 19 pulsars,

- given the glitch size and rate distributions as in Fuentes et al. (2017), there are not enough unseen glitches prior to 

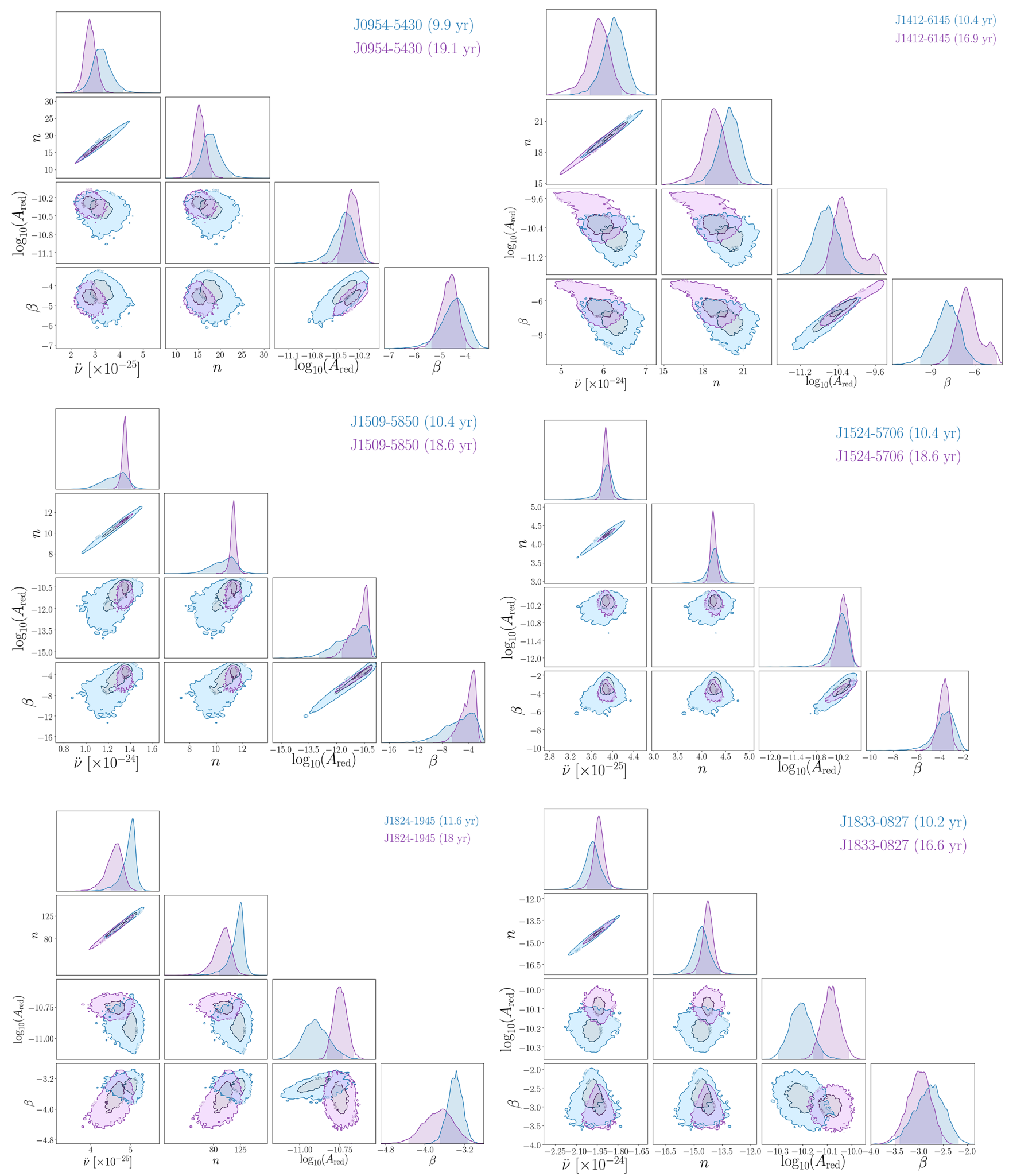

Figure 5. Posterior distributions of six pulsars comparing the original (in blue) and the longer (legacy) data sets (in purple). We account for a previously published glitch at MJD 51868 for PSR J1412-6145. 


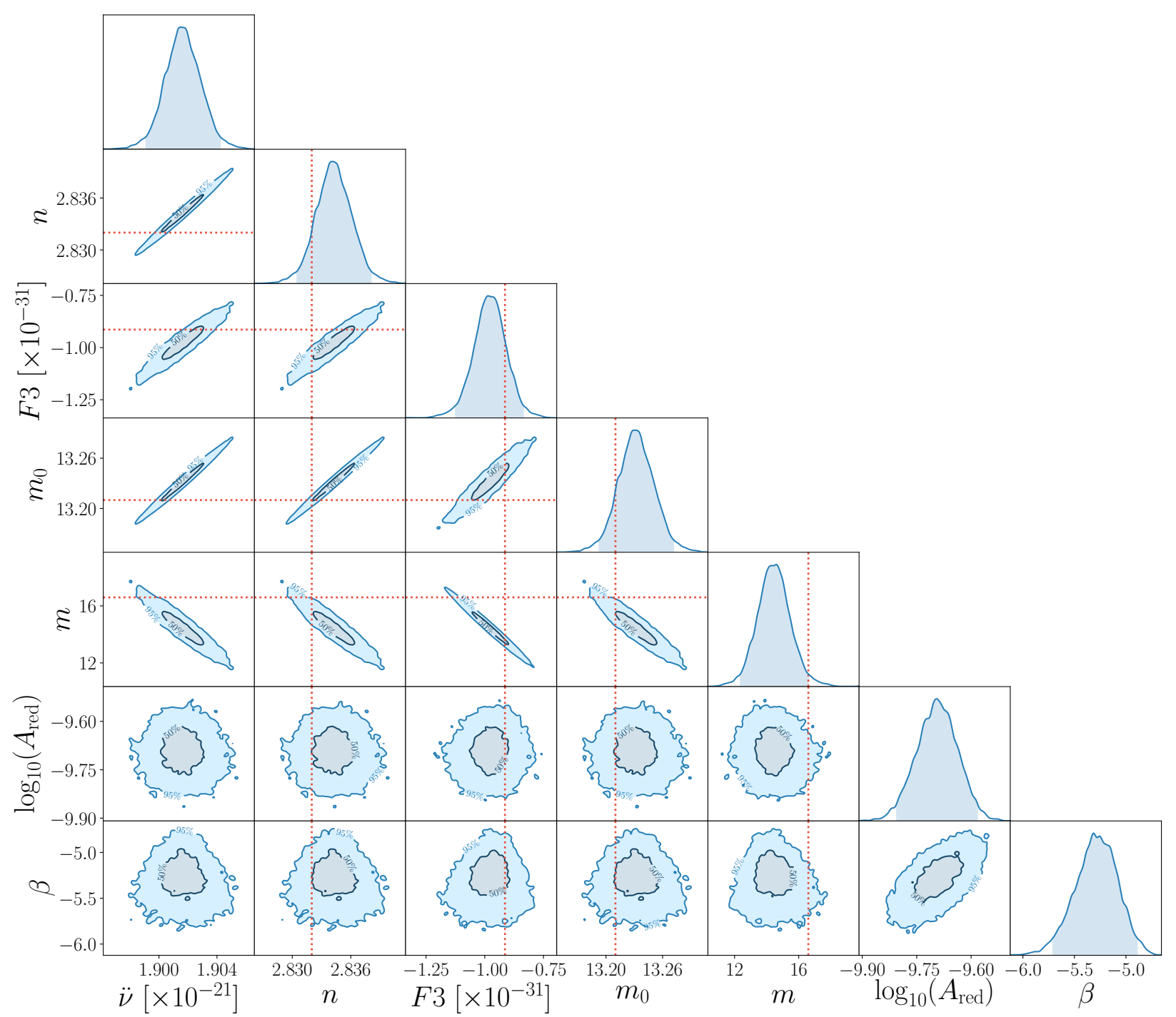

Figure 6. Posterior distributions of $\ddot{v}, \ddot{v}, n, m_{0}, m$ and the timing noise parameters for PSR J1513-5908 measured from 25.4 years of radio timing data. The dashed orange lines are measurements from Livingstone \& Kaspi (2011).

the beginning of our dataset to explain the values of $n$ we obtain even for long recovery times,

- for long exponential glitch recovery times comparable to our data span, TEMPONEST will detect and distinguish the presence of a glitch recovery signal from a cubic term.

In conclusion, there is strong evidence for a cubic term in the residuals of the 19 pulsars rather than an exponential term and the measured values of braking indices are unlikely due to an exponential glitch recovery.

\section{IMPLICATIONS}

In Figure 9, we show evolutionary tracks for each of our pulsars given their measured braking index. From equation 1 , if $K$ and $n$ are assumed to be constant in time, then the pulsars follow a track with slope $2-n$ in the $P-\dot{P}$ diagram. So, for pure magnetic braking with a constant dipolar magnetic field, we expect neutron stars to follow lines with slope of -1 . However, previously measured values of $n$ have been different from the expected value of 3 and in this paper we have reported $n \gg 3$, implying that neutron stars can exhibit substantial torque decay and that the simple dipolar spin-down model is clearly imperfect.

We find evidence for a mild correlation $(r=0.34 \pm 0.01)$ between the characteristic age and $n$ as shown in Figure 10. The correlation coefficient is computed based on the Spearmann correlation test, which is typically robust to outliers. PSR J1513-5908 is the youngest pulsar in our sample with a characteristic age of $1.6 \mathrm{kyr}$, while PSR J1824-1945 has a characteristic age of $570 \mathrm{kyr}$. PSR J0857-4424 has the highest measured $n$ which is clearly an outlier for its characteristic age; this is further discussed in Section 6.3. In PSJ19, we 

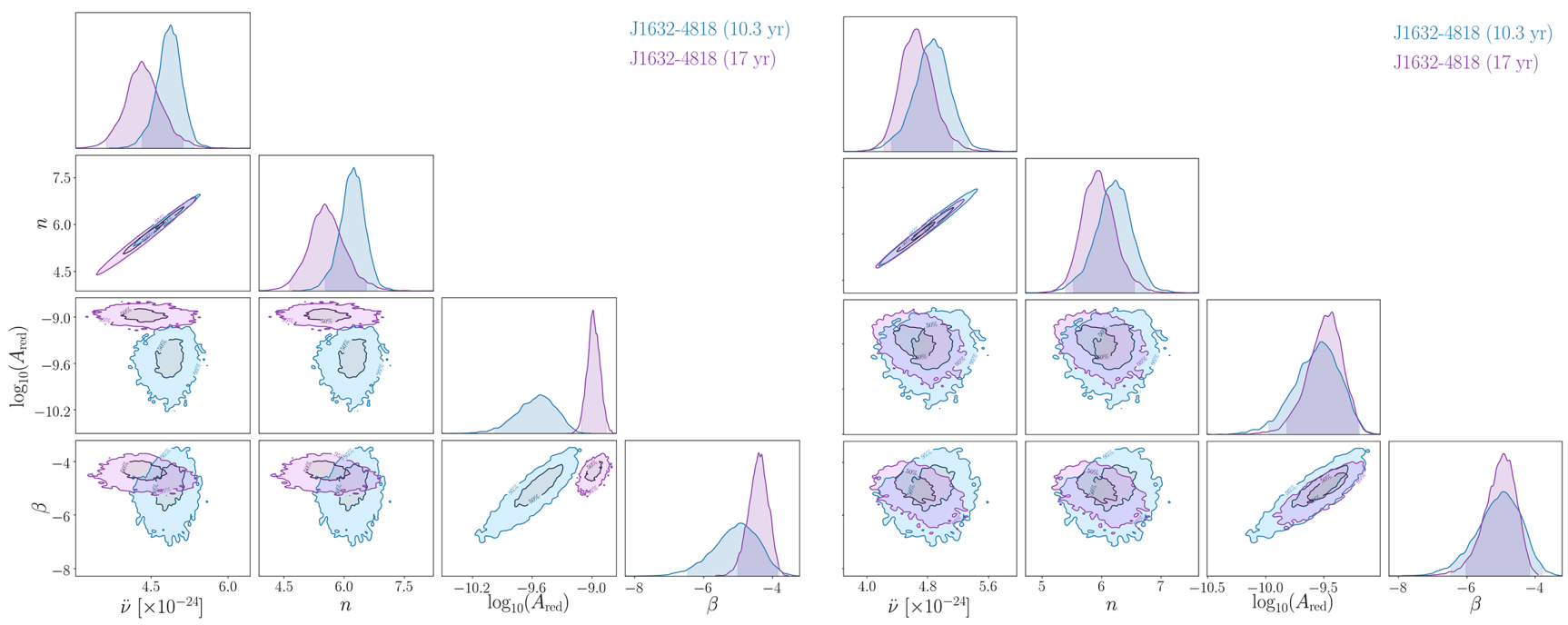

Figure 7. Posterior distributions for PSR J1632-4818, for the PSJ19 (in blue) and legacy data sets (in purple) before (left) and after (right) modelling the glitch.
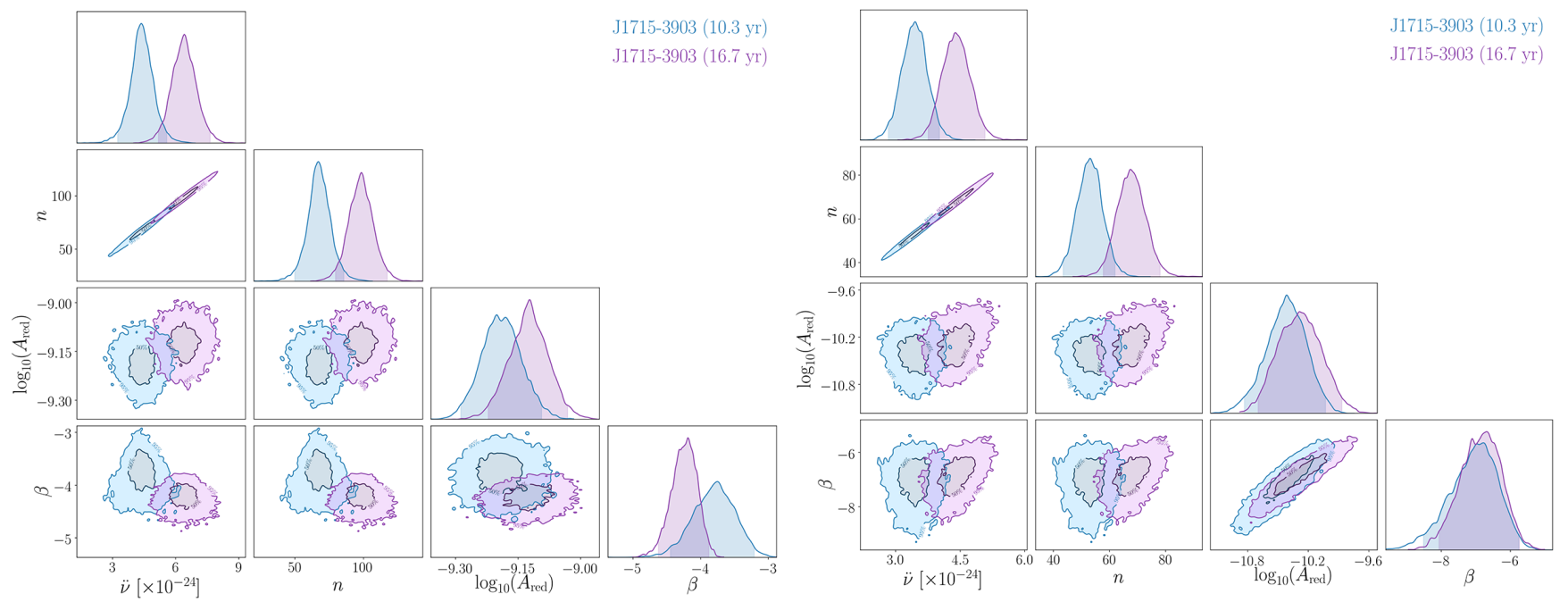

Figure 8. Posterior distributions for PSR J1715-3903, for the PSJ19 (in blue) and legacy (in purple) data sets before (left) and after (right) modelling the glitch.

reported limits on $n$ for pulsars for which the $\ddot{v}$ model was not preferred. Adding pulsars from PSJ19 with the most constrained upper limits on $n$ (represented by blue circles) does not affect this correlation. If we consider a line of constant age drawn at $\tau_{\mathrm{c}} \sim 120 \mathrm{kyr}$ in Figure 9, we detect $n$ in 12 out of the 19 younger pulsars as opposed to detecting $n$ in only 7 out of 66 pulsars with $\tau_{\mathrm{c}}>120$ kyr. Two questions thus arise, first why do we measure significant braking indices in the younger pulsars and secondly, how can we connect the short-term braking values with the long-term evolutionary tracks in $P-\dot{P}$ space?

\subsection{The detectability of braking index}

To address the first question posed above, we discuss the various factors that play a role in measuring a braking index. The detectability of $n$ for a pulsar depends on, $v, \dot{v}, \ddot{v}, T_{\text {span }}$, the mean red-noise amplitude $\left(A_{\text {red }}(\mathrm{TN})\right)$ and the spectral index $(\beta(T N))$ of the timing noise.

Consider the residuals, $R(t)$, induced through a $\ddot{v}$ :

$R_{\ddot{v}}(t)=\frac{1}{6}\left(\frac{\ddot{v}}{v}\right) t^{3}$,

The residuals are sampled at equal intervals over a time span $-T / 2<t<T / 2$. The variance of these residuals is then

$\sigma_{\ddot{v}}^{2}(T)=\frac{1}{16128}\left(\frac{\ddot{v}}{v}\right)^{2} T^{6}$ 


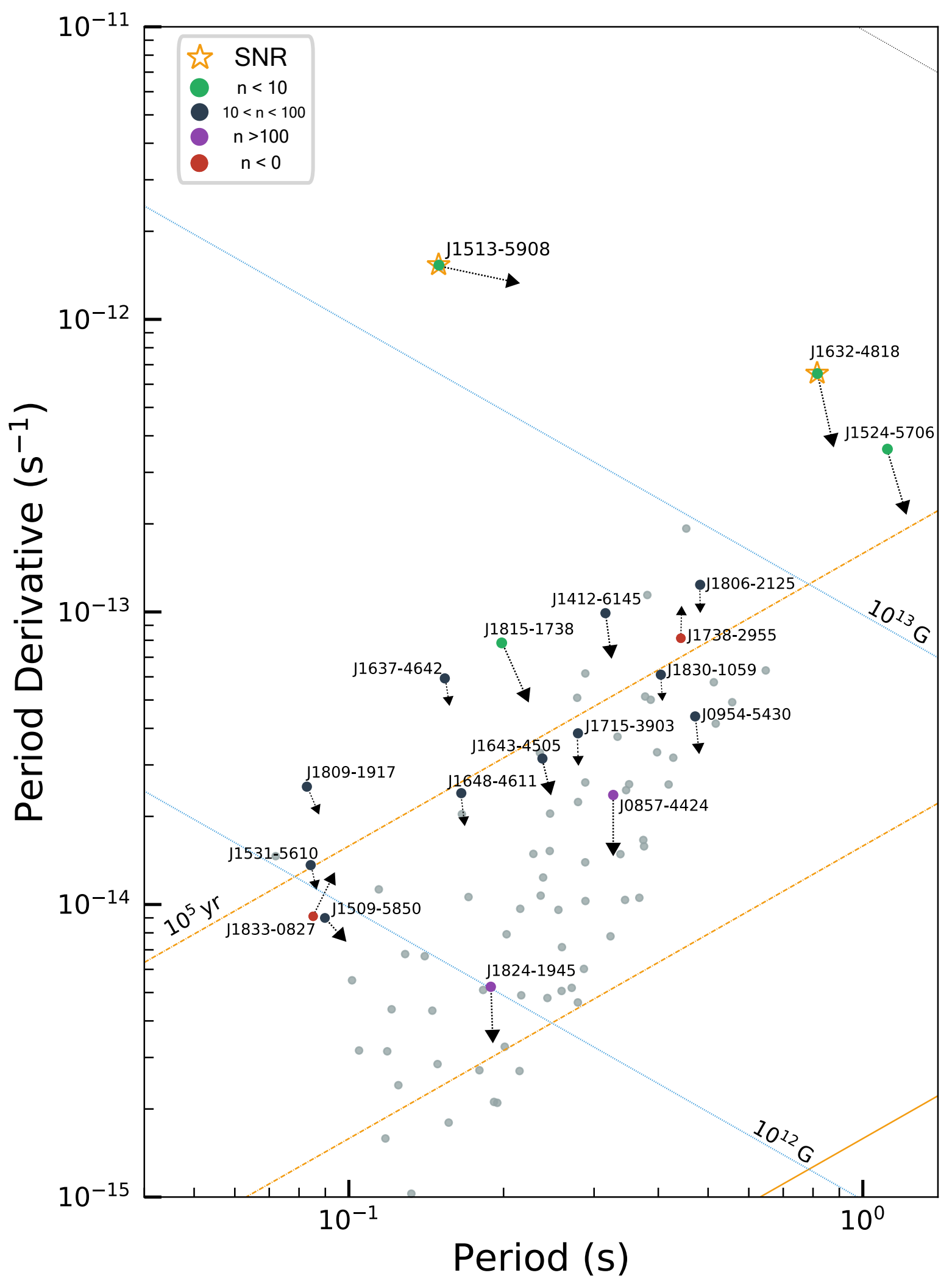

Figure 9. The $P-\dot{P}$ diagram for the 19 pulsars discussed in this paper (colour-coded filled circles) and the rest of the pulsars discussed in PSJ19 (grey circles). Lines of constant age are in orange (dashed line), while lines of constant magnetic fields are in blue. The dashed arrows show the time-evolution of the pulsars which follow a slope of $2-n$. The length of the arrows are arbitrary to preserve clarity. 


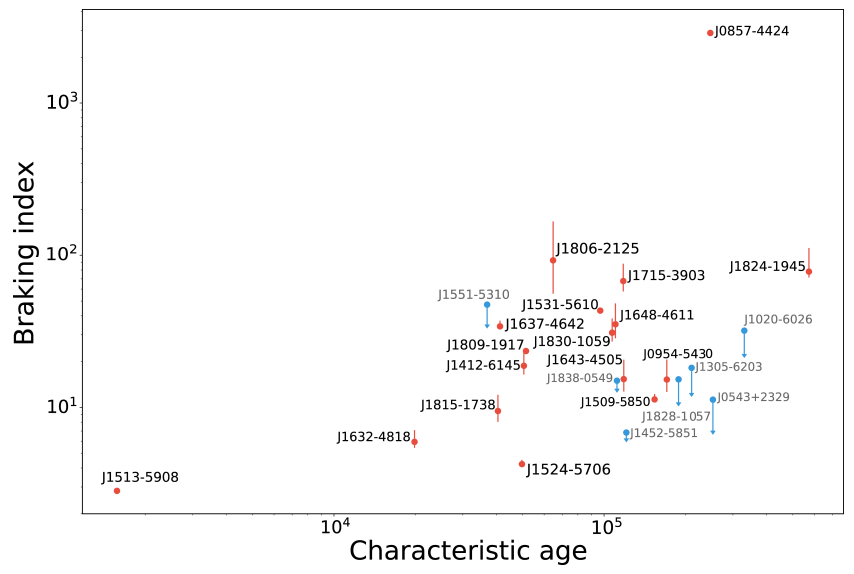

Figure 10. Characteristic age $\left(\tau_{\mathrm{c}}\right)$ versus braking index for 17 pulsars discussed in this paper (red circles) along with seven nondetections of $n$ (from PSJ19) with strongly constrained limits (blue circles). The uncertainties on the red circles are $97.5 \%$ and $2.5 \%$ confidence limits. The pulsars with negative values of $n$, PSRs J1738-2955 and J1833-0827 are not included in this figure. They have characteristic ages of $85 \mathrm{kyr}$ and $147 \mathrm{kyr}$ respectively. is

The variance of a power law, expressed as $P(f)=A f^{\beta}$

$$
\begin{aligned}
\sigma_{\mathrm{PL}}^{2} & =\int_{1 / T}^{\infty} d f A f^{\beta} \\
& =\left.\frac{A}{\beta+1} f^{\beta+1}\right|_{1 / T} ^{\infty}, \\
& =-\frac{A}{\beta+1} T^{-\beta-1}
\end{aligned}
$$

where $A$ is the amplitude and $\beta$ is the spectral index. Relating equations 11 and 12 , and expressing $\ddot{v}$ in terms of $n$ (from equation 2) we derive an expression for the power spectral density associated with $n$

$P_{n}(f)=\frac{n^{2}}{2688}\left(\frac{\dot{v}}{v}\right)^{4} f^{-7}$

This can be used to assess the detectability of a cubic in the presence of other noise sources in the timing residuals. White noise, arising largely due to measurement errors, has a flat spectrum and if the amplitude of the cubic is higher than the white noise level, it will be detectable. Timing noise is a red noise process and is modelled as a power law process with a spectral index $\beta$ (see equation 4 ). Considering equation 13 we can see that:

- the power spectral density of the cubic is larger in pulsars with smaller characteristic ages (Espinoza et al. 2017),

- in pulsars where the spectral index of the timing noise is $\beta>-7$, the cubic spectral power will dominate over the timing noise for large time intervals,

- in pulsars where $\beta<-7$ (steep timing noise) the timing noise will dominate the cubic even with long observing time spans.

The S/N $(S)$ of a deterministic signal $(r)$ characterized by a noise covariance matrix $(C)$ is expressed as,

$S=\sqrt{\boldsymbol{r}^{\dagger} \mathbf{C}^{-1} \boldsymbol{r}}$.

The covariance matrix $(C)$ describes the contributions from stochastic red noise and white noise components. A cubic signal is likely to be detected when $S \gg 1$. As the parameters describing the red noise process are measured directly from the data, there is an inherent degeneracy between the noise covariance matrix and the signal, which is not taken into account in this methodology. In the frequency domain, the noise covariance matrix is diagonal $\left(C_{i j}=0\right.$ for $\left.i \neq j\right)$ and is expressed as,

$C_{i i}=\left(A f_{i}^{\beta}+\frac{2 T \sigma_{W N}^{2}}{N}\right)$

where $\sigma_{W N}$ is the variance of the white noise components and is assumed to be equal in all observations.

Using the noise covariance matrix and the signal, a detection statistic can be calculated to determine the $\mathrm{S} / \mathrm{N}$ of a cubic in the presence of white and red noise components,

$S^{2}=\sum_{i} r_{i}^{\dagger} r_{i} C_{i i}^{-1}=\left(\frac{n^{2}}{2688}\right)\left(\frac{\dot{v}}{v}\right)^{4} \sum_{i} \frac{f_{i}^{-7}}{A f_{i}^{\beta}+\left(2 T \sigma_{W N}^{2} / N\right)}$

Using the red noise models, the time $T$ and the $\sigma_{\mathrm{WN}}$ for each of the pulsars in PSJ19, we can compute a $S_{3}$ based on equation 16 for $n=3$. For 15 of the 19 pulsars with measured braking indices, we find that $S_{3} \gg 1$, giving a good indication of the usefulness of the metric. The remaining four pulsars have large values of $n$ which when scaled accordingly will boost the value of $S_{3}$. Pulsars for which we do not detect a significant value of $n$ but have $S_{3}>1$ seem to favour a model with low-frequency components. The youngest pulsar in our sample without a measured $n$ is PSR J1551-5310. It has $S_{3}=0.9$ due to a combination of dominant timing noise $(\beta=-7.3)$ and a relatively short timing baseline of $\sim$ 7 years. The value of $S_{3}$ increases with time in those pulsars with shallow timing noise, hence we expect to detect more braking indices as our time span increases.

\subsection{Pulsar evolution in the $P-\dot{P}$ diagram.}

For PSR J1513-5908 we stated that since our measurement of the second braking index $(m)$ is consistent with the expected value from the standard spin-down model, it is reasonable to assume that $n$ has not yet evolved with time. It is thus interesting to speculate on the future evolution of PSR J1513-5908. If its braking index remains constant, the evolutionary track will take it above the bulk of the population in the $P-\dot{P}$ plane. It will cross the radio death-line with a period of $\sim 7 \mathrm{~s}$, and perhaps then occupy the part of the $P$ $\dot{P}$ diagram containing the X-ray dim isolated neutron stars (Turolla 2009; Rigoselli et al. 2019). On the other hand its braking index could increase with time, and bring it close to PSR J1632-4818 in 34 kyr, eventually following evolutionary tracks characterised by larger values of $n$ to become a $\sim 1$ s pulsar with ever decreasing $\dot{P}$.

PSRs J1738-2955 and J1833-0827 have a negative braking index implying that they evolve upwards and to the right in $P-\dot{P}$ space. A similar upward evolution is seen for PSR $\mathrm{J} 1734-3333$, which has a measured $n$ of $0.9 \pm 0.2$ (Espinoza et al. 2011b) and is speculated to attain the rotational properties of a magnetar in $\sim 30 \mathrm{kyr}$ as a consequence of surface magnetic field growth. PSR J1833-0827 is at a distance of $\sim 4.3 \mathrm{kpc}$, estimated both from DM (Yao et al. 2017) and 
HI absorption measurements (Weisberg et al. 1995), and has been plausibly associated with the SNR W41 (Gaensler \& Johnston 1995). Our transverse velocity measurement of $800 \pm 200 \mathrm{~km} \mathrm{~s}^{-1}$, suggests that the pulsar is indeed moving away from the SNR. We speculate that the magnetic field of the pulsar could still be increasing, thus causing the negative braking index. Another possibility is that the geometry of this pulsar is evolving towards orthogonality as is the case for the Crab pulsar (Lyne et al. (2013)). The dense plasma from the pulsar wind nebula surrounding this pulsar (Esposito et al. 2011) could exert a retardation torque on the neutron star causing a change in $\alpha$ (Beskin \& Nokhrina 2007). Constraints on the viewing geometry and its time dependence could prove fruitful.

What then is the link between the high values of $n$ measured here over $\sim 15$ year time-scales with the evolution over timescales of Myr? We know of pulsars with similar characteristics to the ones discussed here, but with higher glitch rates. The spin-down of these pulsars is dominated by large braking indices $(n>50)$ between the glitches followed by a "reset" of the $\dot{v}$ at the glitch itself (Yu et al. 2013, Espinoza et al. 2017). Espinoza et al. (2017) therefore argue that longterm value of $n$ is indeed close to the canonical value of 3 . Could it be then, that in our sample of pulsars, we are measuring $n$ in between glitches separated by $>20$ yr and that a future glitch will reset the $\dot{v}$ back to its expected value from $n=3$ ? Based on the theoretical work of Alpar et al. (1993) and Alpar \& Baykal (2006) who postulated a weak coupling between the crust of the star and the superfluid interior, Yu et al. (2013) demonstrated an observational relationship describing the inter-glitch value of $\ddot{v}_{g}$ :

$\ddot{v}_{g}=10^{-2.8 \pm 1.4} \dot{v} / T_{g}$

where $T_{g}$ is the mean time between glitches. In turn, Fuentes et al. (2017) showed that $T_{g} \propto \dot{v}^{-1}$. Combining this with equation 2 , yields the remarkable relationship for the braking index between glitches, $n_{g}$, which then depends only on $v$,

$n_{g}=10^{-0.2 \pm 1.4} v$.

We do not find a good correlation between $n$ and $v$ in our data, a formal fit yields $n_{g}=10^{+0.3} v$ but with a large scatter. We therefore surmise that our values of $n$ contain an "intrinsic" value higher than 3 coupled with an inter-glitch value. We also note that if the glitch resets the evolutionary track back to a slope described by $n=3$ for long glitch intervals, then this implies that the change in $\Delta \dot{v} / \dot{v}$ must be larger than $10^{-2}$ in this set of pulsars. Therefore, there should be a correlation between $\Delta \dot{v} / \dot{v}$ and the waiting time between glitches. However, current data is too sparse to determine this. In any case, the evidence remains strong for $n$ to increase in the long-term in order to populate the $P-\dot{P}$ plane with the known pulsar population, either due to the alignment of the magnetic and rotational axes of the pulsar (Johnston \& Karastergiou 2017) or magnetic field decay (Viganò et al. 2013).

If the braking index is indeed increasing with age, then it could imply that older pulsars have smaller inclination angles, as proposed by Tauris \& Manchester (1998), Weltevrede \& Johnston (2008), Johnston \& Karastergiou (2019). Measuring the pulsar's geometry would then help to confirm this idea. For the 19 pulsars in this sample, only three pulsars have been subject to geometrical analysis (PSRs J1513-5908, J1531-5610 and J1648-4611), and even these are poorly constrained (Rookyard et al. 2015). Comparisons between $n$ and $\alpha$ for a large sample of young pulsars will be an important test to further constrain theoretical predictions.

\subsection{A wide-orbit companion for PSR J0857-4424?}

A gravitational interaction between a pulsar and a companion in a wide orbit can manifest itself as the second derivative of the pulsar spin period (Matthews et al. 2016; Kaplan et al. 2016; Bassa et al. 2016). This can be expressed as (from Kaplan et al. 2016),

$\ddot{P}=\frac{G^{1 / 3} M_{\mathrm{c}} P \sin i}{\left(M_{\mathrm{c}}+M_{\mathrm{psr}}\right)^{2 / 3} c}\left(\frac{2 \pi}{P_{\mathrm{b}}}\right)^{7 / 3} \cos \phi$,

where $G$ is the gravitational constant, $c$ is the speed of light, $M_{\mathrm{psr}}$ is the mass of the pulsar, $M_{\mathrm{c}}$ is the mass of the orbiting companion, $P$ is the pulsar's spin period, $i$ is the orbital inclination angle, $a$ is the orbital semi-major axis and $\phi$ is the orbital phase. If we attribute the entirety of the measured $\ddot{P}$ to this effect then the orbital period $\left(P_{\mathrm{b}}\right)$ is given by

$P_{\mathrm{b}}=\left(\frac{M_{\mathrm{c}} \cos \phi \sin i G^{1 / 3} P 2 \pi^{7 / 3}}{\left(M_{\mathrm{c}}+M_{\mathrm{psr}}\right)^{2 / 3} \ddot{P}}\right)^{3 / 7}$,

For simplicity, if we assume a circular orbit, with $M_{\mathrm{psr}}$ of $1.44 M_{\odot}$ and $\sin i=0.5$, and additionally set $\cos \phi=1$, we can derive an empirical relation between $M_{\mathrm{c}}$ and the maximum $P_{\mathrm{b}}$ for a range of companion masses.

We consider the case for PSR J0857-4424, the pulsar with the highest value of $n$ in our sample. We take companion masses of $0.1,1$ and $10 M_{\odot}$ and compute $P_{\mathrm{b}}$ using equation 20. Table 5 reports the computed orbital periods, and the corresponding orbital separation for various companion masses. The estimated distance to PSR J0857-4424 from the DM measurement is $\sim 2.8 \mathrm{kpc}$. At this distance, a $0.1 M_{\odot}$ main-sequence star will have an apparent magnitude of $\sim 29$, while $1 M_{\odot}$ and $10 M_{\odot}$ main-sequence stars will have apparent magnitudes of $\sim 17$ and $\sim 11$ respectively. We examined the Gaia database for stars within a radius of 10 arcsec from the pulsar position and found no objects (Lindegren et al. 2018). Since the limiting magnitude for Gaia is 20 , we can place an upper limit of $\sim 0.8 M_{\odot}$ on a main sequence companion. However, if the companion is a $1 M_{\odot}$ white dwarf or a neutron star, it would not be detected by Gaia.

It is hard to determine the likelihood of such a wide binary system existing. While we know at least one system with an orbital period in excess of $50 \mathrm{yr}$ (Lyne et al. 2015b, $\mathrm{Ng}$ et al. 2019), the companion is a $15 M_{\odot}$ main sequence star easily seen in the optical. At least one millisecond pulsar (Thorsett et al. 1993) appears to have a low-mass companion in a very long orbit but this system is in a globular cluster which permits such exotic formation scenarios. Therefore we entertain a binary orbit as a potential explanation of the high braking index of PSR J0857-4424 albeit an unlikely one. 
Table 5. Estimated upper limits the orbital periods and orbital separations for three different companion masses for PSR J08574424 computed using equation 20.

\begin{tabular}{lrr}
\hline \hline $\begin{array}{l}\text { Companion mass } \\
\left(\boldsymbol{M}_{\odot}\right)\end{array}$ & $\begin{array}{r}\text { Orbital period } \\
(\mathrm{yr})\end{array}$ & $\begin{array}{r}\text { Orbital separation } \\
(\mathrm{AU})\end{array}$ \\
\hline 0.1 & 60 & 20 \\
1 & 130 & 35 \\
10 & 230 & 85 \\
\hline
\end{tabular}

\section{CONCLUSIONS}

There are several strands to the work presented here. First, we restate the important point that implementing a full timing model, which includes timing noise parameterization into a Bayesian framework like TEMPONEST, means that models can be evaluated and compared based on their Bayesian evidence. This is not possible in the generalized least-squares method implemented in TEMPO2. We have shown that the values of $n$ derived in PSJ19 are robust to the addition of historical data, which emphasizes that they are unlikely to be due to unseen exponential glitches in the past. Even in the presence of glitches, successful detection and modelling of the glitch parameters maintains the consistency of $n$ across long timing baselines. Furthermore, we show that we can distinguish between models with and without an exponential glitch recovery signal and that any unseen glitch prior to our data commencing, must have little or no influence on the results presented here either because (a) the glitch size is small and/or (b) that the glitch decay time is less than 1000 days. This is supported by glitch statistics which inform that it is highly unlikely for all the pulsars presented here to have suffered large enough glitches in the immediate past to cause the presence of an exponential signal in the data.

We are therefore left with the conclusion that the large values of $n$ measured here are indeed indicative of the way a pulsar spins down, at least over a timescale of a decade or more. We consider it likely that $n$ gets reset at the time of a glitch and that the inter-glitch value of $n$ can be large. We predict a correlation between the wait time between glitches and the value of the step in $\dot{v}$.

Based on these results, we discuss the implications of the evolution of pulsars in the $P-\dot{P}$ diagram and find a moderate correlation of braking index with characteristic age, which can be attributed to either magnetic field decay and/or changes in the pulsar's inclination angle. We discuss an evolutionary path for PSR J1513-5908 depending on the future behaviour of its braking torque and speculate on the effect of a dense PWN nebula surrounding PSR J18330827 in imparting it with a negative braking index. We outline a metric to compute the detectability of $n$ in the presence of timing noise. Finally we explore the possibility of a wide-orbit companion inducing a very high braking index in PSR J0857-4424.

\section{ACKNOWLEDGEMENTS}

The Parkes radio telescope is part of the Australia Telescope, which is funded by the Commonwealth Government for op- eration as a National Facility managed by CSIRO. This work made use of the gSTAR and OzSTAR national HPC facilities. gSTAR is funded by Swinburne and the Australian Government Education Investment Fund. OzSTAR is funded by Swinburne and the National Collaborative Research Infrastructure Strategy (NCRIS). This work is supported through Australian Research Council (ARC) Centre of Excellence CE170100004. A.P thanks Chris Flynn for help with the Gaia analysis and George Hobbs for stimulating discussions on glitches and timing noise. A.P. acknowledges support from CSIRO Astronomy and Space Science. R.M.S. acknowledges support through ARC grant CE170100004. M.B, S.O, and R.M.S. acknowledge support through ARC grant FL150100148. Work at NRL is supported by NASA. This work also made use of standard Python packages (Oliphant 2006, Virtanen et al. 2020, McKinney 2010, Hunter 2007), Chainconsumer (Hinton 2016) and Bokeh (Bokeh Development Team 2018).

\section{REFERENCES}

Akbal O., Alpar M. A., Buchner S., Pines D., 2017, MNRAS, 469, 4183

Alpar M. A., Baykal A., 2006, MNRAS, 372, 489

Alpar M. A., Chau H. F., Cheng K. S., Pines D., 1993, ApJ, 409, 345

Anderson P. W., Itoh N., 1975, Nature, 256, 25

Andersson N., Antonopoulou D., Espinoza C. M., Haskell B., Ho W. C. G., 2018, ApJ, 864, 137

Antonopoulou D., Espinoza C. M., Kuiper L., Andersson N., 2018, MNRAS, 473, 1644

Archibald R. F., et al., 2016, ApJ, 819, L16

Bassa C. G., et al., 2016, MNRAS, 460, 2207

Baym G., Pethick C., Pines D., 1969, Nature, 224, 673

Beskin V. S., Nokhrina E. E., 2007, Ap\&SS, 308, 569

Blandford R. D., Romani R. W., 1988, MNRAS, 234, 57P

Blandford R. D., Applegate J. H., Hernquist L., 1983, MNRAS, 204, 1025

Bokeh Development Team 2018, Bokeh: Python library for interactive visualization. https://bokeh.pydata.org/en/latest/

Candy B. N., Blair D. G., 1986, ApJ, 307, 535

Cheng K. S., 1987, ApJ, 321, 805

Cole T. W., 1969, Nature, 223, 487

Coles W., Hobbs G., Champion D. J., Manchester R. N., Verbiest J. P. W., 2011, MNRAS, 418, 561

Davies J. G., Hunt G. C., Smith F. G., 1969, Nature, 221, 27

Espinoza C. M., Lyne A. G., Stappers B. W., Kramer M., 2011a, MNRAS, 414, 1679

Espinoza C. M., Lyne A. G., Kramer M., Manchester R. N., Kaspi V. M., 2011b, ApJ, 741, L13

Espinoza C. M., Lyne A. G., Stappers B. W., 2017, MNRAS, 466, 147

Esposito P., et al., 2011, MNRAS, 416, 205

Ferdman R. D., Archibald R. F., Gourgouliatos K. N., Kaspi V. M., 2018, ApJ, 852, 123

Fuentes J. R., Espinoza C. M., Reisenegger A., Shaw B., Stappers B. W., Lyne A. G., 2017, A\&A, 608, A131

Gaensler B. M., Johnston S., 1995, MNRAS, 275, L73

Groth E. J., 1975, ApJSS, 29, 453

Gunn J. E., Ostriker J. P., 1969, Nature, 221, 454

Harding A. K., Contopoulos I., Kazanas D., 1999, ApJ, 525, L125

Haskell B., Melatos A., 2015, International Journal of Modern Physics D, 24, 1530008

Hinton S. R., 2016, The Journal of Open Source Software, 1, 00045

Ho W. C. G., Andersson N., 2012, Nature Physics, 8, 787 
Hobbs G., Lyne A. G., Kramer M., Martin C. E., Jordan C., 2004, MNRAS, 353, 1311

Hobbs G., Lyne A. G., Kramer M., 2010, MNRAS, 402, 1027

Hunter J. D., 2007, Computing In Science \& Engineering, 9, 90

Johnston S., Galloway D., 1999, MNRAS, 306, L50

Johnston S., Karastergiou A., 2017, MNRAS, 467, 3493

Johnston S., Karastergiou A., 2019, MNRAS, 485, 640

Jones P. B., 1990, MNRAS, 246, 364

Kaplan D. L., et al., 2016, ApJ, 826, 86

Kaspi V. M., Manchester R. N., Siegman B., Johnston S., Lyne A. G., 1994, ApJ, 422, L83

Kramer M., Lyne A. G., O'Brien J. T., Jordan C. A., Lorimer D. R., 2006, Science, 312, 549

Lentati L., Alexander P., Hobson M. P., Feroz F., van Haasteren R., Lee K. J., Shannon R. M., 2014, MNRAS, 437, 3004

Lindegren L., et al., 2018, A\&A, 616, A2

Livingstone M. A., Kaspi V. M., 2011, ApJ, 742, 31

Livingstone M. A., Kaspi V. M., Gavriil F. P., Manchester R. N., 2005, ApJ, 619, 1046

Lyne A., Hobbs G., Kramer M., Stairs I., Stappers B., 2010, Science, 329, 408

Lyne A., Graham-Smith F., Weltevrede P., Jordan C., Stappers B., Bassa C., Kramer M., 2013, Science, 342, 598

Lyne A. G., Jordan C. A., Graham-Smith F., Espinoza C. M., Stappers B. W., Weltevrede P., 2015a, MNRAS, 446, 857

Lyne A. G., Stappers B. W., Keith M. J., Ray P. S., Kerr M., Camilo F., Johnson T. J., 2015b, MNRAS, 451, 581

Manchester R. N., Durdin J. M., Newton L. M., 1985, Nature, 313,374

Marshall F. E., Guillemot L., Harding A. K., Martin P., Smith D. A., 2016, ApJ, 827, L39

Matthews A. M., et al., 2016, ApJ, 818, 92

McKinney J. C., 2006, MNRAS, 368, L30

McKinney W., 2010, in van der Walt S., Millman J., eds, Proceedings of the 9th Python in Science Conference. pp $51-$ 56

Melatos A., Warszawski L., 2009, ApJ, 700, 1524

Middleditch J., Marshall F. E., Wang Q. D., Gotthelf E. V., Zhang W., 2006, ApJ, 652, 1531

Namkham N., Jaroenjittichai P., Johnston S., 2019, MNRAS, 487, 5854

Ng C. Y., et al., 2019, ApJ, 880, 147

Oliphant T., 2006, NumPy: A guide to NumPy, USA: Trelgol Publishing

Parthasarathy A., et al., 2019, MNRAS, 489, 3810

Rigoselli M., Mereghetti S., Suleimanov V., Potekhin A. Y., Turolla R., Taverna R., Pintore F., 2019, A\&A, 627, A69

Rookyard S. C., Weltevrede P., Johnston S., 2015, MNRAS, 446, 3367

Shannon R. M., Cordes J. M., 2010, ApJ, 725, 1607

Shannon R. M., Lentati L. T., Kerr M., Johnston S., Hobbs G., Manchester R. N., 2016, MNRAS, 459, 3104

Spitkovsky A., 2006, ApJ, 648, L51

Tauris T. M., Konar S., 2001, A\&A, 376, 543

Tauris T. M., Manchester R. N., 1998, MNRAS, 298, 625

Thorsett S. E., Arzoumanian Z., Taylor J. H., 1993, ApJ, 412, L33

Turolla R., 2009, in Becker W., ed., Vol. 357, Astrophysics and Space Science Library. p. 141, doi:10.1007/978-3-540-76965$1 \_7$

Viganò D., Rea N., Pons J. A., Perna R., Aguilera D. N., Miralles J. A., 2013, MNRAS, 434, 123

Virtanen P., et al., 2020, Nature Methods, 17, 261

Weisberg J. M., Siegel M. H., Frail D. A., Johnston S., 1995, ApJ, 447, 204

Weltevrede P., Johnston S., 2008, MNRAS, 387, 1755

Yao J. M., Manchester R. N., Wang N., 2017, ApJ, 835, 29
Young M. D. T., Chan L. S., Burman R. R., Blair D. G., 2010, MNRAS, 402, 1317

Yu M., et al., 2013, MNRAS, 429, 688

van Haasteren R., Levin Y., 2013, MNRAS, 428, 1147

This paper has been typeset from a $\mathrm{TEX}_{\mathrm{E}} \mathrm{LATEX}$ file prepared by the author. 